\title{
Peak-to-Average Power Ratio Reduction Using Active Constellation Extension-Projection Onto Convex Sets Combined with Particle Swarm Optimization Added to Space Time Bloc Coding in Multiple Input Multiple Output-Orthogonal Frequency Division Multiplexing System
}

\author{
${ }^{1}$ Ahmed Ouqour, ${ }^{2}$ Abdelhamid Louliej, \\ ${ }^{2}$ Younes Jabrane, ${ }^{1}$ Brahim Ait Es Said and ${ }^{2}$ Abdellah Ait Ouahman \\ ${ }^{1}$ Department of Telecommunications and Networks, \\ Faculty of Sciences Semlalia, Cadi Ayyad University, Marrakesh, Morocco \\ ${ }^{2}$ Department of Telecom Engineering, \\ National School of Applied Sciences, Cadi Ayyad University, Marrakesh, Morocco
}

Received 2012-09-06, Revised 2012-09-30; Accepted 2012-12-11

\begin{abstract}
When generated Orthogonal Frequency Division Multiplexing (OFDM) signal is transmitted through a number of antennas in order to achieve diversity or cap any gain (higher transmission rate) then it is known as Multiple Input Multiple Output (MIMO)-OFDM. The full form of MIMO is multi input and multi output where signals are transmitted via multiple antennas instead of only one antenna like FDM. This technique has the potential of dramatic increase of data transmission in wireless environment. However, the transmitted signal still has high Peak-to-Average Power Ratio (PAPR) because of OFDM characteristics. The proposal algorithm in this study describes a new technique using Active Constellation Extension-Projection Onto Convex Sets (ACE-POCS) combined with the Particle Swarm Optimization (PSO) and Space Time Bloc Coding (STBC) for PAPR reduction. Apply the ACE-POCS technique and independently on each transmitted antenna, was effective to reduce PAPR, but converges slowly. Therefore, the Particle Swarm Optimization (PSO) was introduced to fight this slowness, by searching and well reducing the PAPR. Thanks of the PSO-ACE-POCS algorithm; the transmitted sequence was selected with best PAPR reduction over all transmission antennas. The results of simulation and BER performance show that the PSO-ACE-POCS method added to STBC minimizes the PAPR with a fast convergence and low computational complexity in comparison with the ACE-POCS method applied to STBC.
\end{abstract}

Keywords: Peak to Average Power Ratio (PAPR), OFDM-MIMO, Single Input Single Output (SISO), ACE-POCS, Particle Swarm Optimization (PSO), Binary Error Rate (BER), High Power Amplifier (HPA)

\section{INTRODUCTION}

Today's communications require sending large volumes of data. Indeed, with the elevated use of wireless LAN technology and systems of third generation mobile telephony, demand for data services has never been greater. The bandwidth of wireless communication systems is generally limited by the cost

Corresponding Author: Ahmed Ouqour, Team of Telecommunications and Networks, Faculty of Science Semlalia, Cadi Ayyad University, Marrakesh, Morocco 
of radio spectrum required. Any increase in bit rate through clever coding techniques and algorithms, makes the system more spectrally efficient and less costly without increasing bandwidth. In this way, traditional wireless communication systems have been made more spectrally efficient. However, the fundamental limitation of bandwidth does not change. To this end, several MIMO communication systems have been an interesting topic for research over the last years, because of their ability to dramatically increase the spectral efficiencies.

As opposed to traditional wireless systems, using one transmitting and one receiving antenna, MIMO systems use arrays of multiple antennas at both ends of the communication link, all operating at the same frequency at the same time. This introduces spatial diversity into the system, which can be used to tackle the problem of multipath.

Otherwise, the OFDM (Beek et al., 1995) is a combination of both modulation and multiplexing. Unlike general multiplexing technique it is applied to only one independent channel, where a set of signals from the same source is divided or spitted into a number of independent channels (sub-set of the main signals) and each modulated by separate carrier and then multiplexed into an OFDM signal for transmission. Each independent sub-set channel can be multiplexed either by frequency or code division multiplexing.

Moreover, each sub-channel of the main signal in fact multiplied by a carrier which is orthogonal to each other. This kind of orthogonality of the carriers allows simultaneous transmission of sub-carriers in a tightly spaced bandwidth without interfering from each other because each carrier is orthogonal to each other making the result of their multiplication a zero. Normally, OFDM signals are sent via one transmitting antenna. Like any other communication systems, MIMO-OFDM (Yang, 2005; Gesbert et al., 2003) system also has transmitter and receiver, but it has multiple antennas both at transmit and receive end. MIMO can be implemented in various ways. If we need to take the diversity as an advantage to combat fading then we need to send the same signals through various MIMO antennas and at the receiving end all the signals received by MIMO antennas will receive the same signals traveled through various path. In this case the entire received signal must pass through uncorrelated channels. Nevertheless, the OFDM signals possess a very wide band and present also a big variation of amplitude. Now, his characteristic is the very sensitive return to the non linearity of the analogical components, in particular the one of the High Power Amplifier (HPA).

In fact, the HPA was conceived to work in his saturation zone (for a yield maximum). Unfortunately, in this zone that presents itself the non-linear harsher. The use of the HPA in his saturation zone provokes the intermodulation, distortions inside the band and ascents of the secondary lob that generate interferences between canals and an increase of the BER.

A several suggestions has been proposed to treat this problem of non linearity, more than a few of them reduces the PAPR and proves itself very effective to relieve overcome problems at the HPA. But, some of these methods treated in (Davis and Jedwab, 1999) are based on coding, in which some bit combinations are sacrificed to eliminate high PAPR patterns while the peak power is reduced as well as the data rate.

Therefore, in this study we exploit PAPR reduction techniques combined to PSO algorithm in (Ouqour et al., 2010; 2011a; Wen et al., 2008), to make an efficient method to reduce PAPR in STBC MIMO-OFDM system with low computational complexity cost.

Hence, we simulate a model of MIMO-OFDM system employing on QPSK and 16-QAM modulations added to STBC using PSO-ACE-POCS algorithm to reduce the PAPR. Then, we evaluate the system performances in terms of BER.

\section{MATERIALS AND METHODS}

\subsection{System Model}

In this study, we explore a MIMO-OFDM system that employs Alamouti (1998) scheme where a STBC is independently applied to each subcarrier (Tsiligkaridis and Jones, 2010). Although several encoding schemes are available for an arbitrary number of transmit antennas, we consider only two transmit antennas for notational simplicity in this study as it shown in Fig. 1 representing the scheme of our system model.

Based on this scheme, we perform a new method using PSO combined with ACE-POCS technique added to STBC to reduce the PAPR of MIMO-OFDM system as well as computational complexity. We will show that our proposal scheme can provide better performance with low complexity in simulation results. So, the main problem in the MIMO-OFDM system is the relatively high PAPR.

Before presenting the proposed method, let's first describe the PAPR calculated relation. 


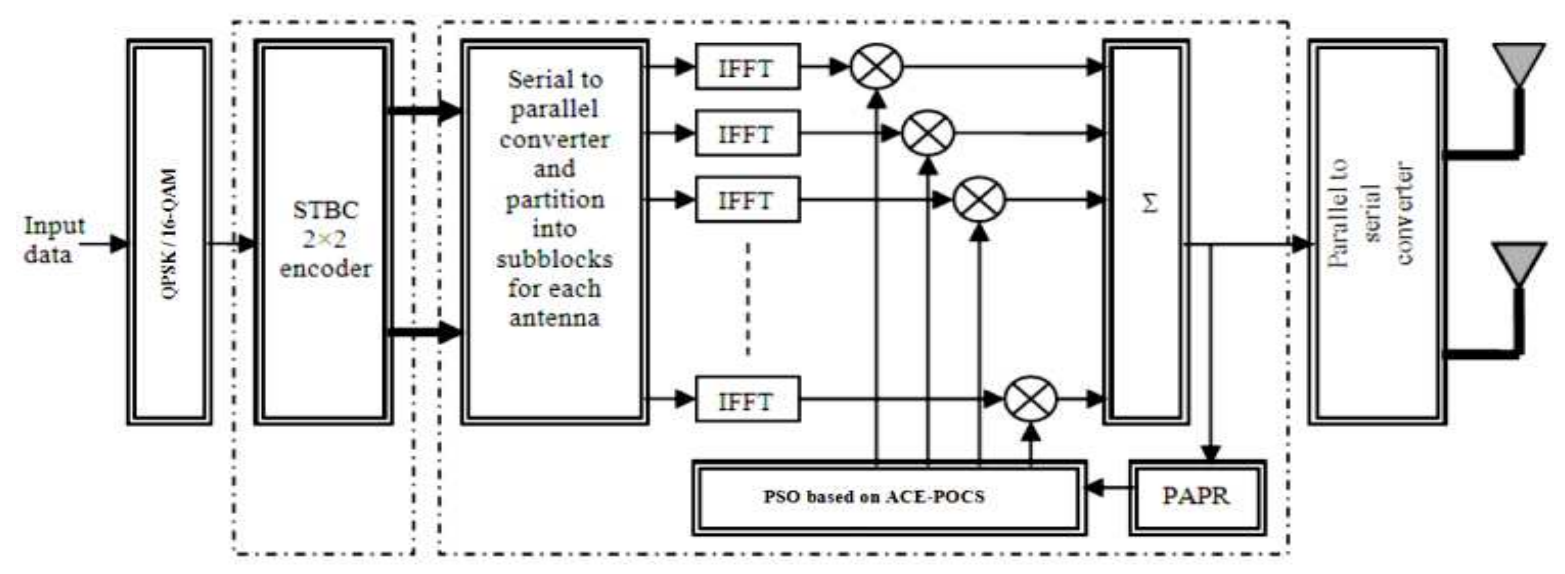

Fig. 1. Scheme of system model

The time-domain complex base-band in a multicarrier system transmitted signal $\mathrm{x}^{\ell}$ for the $\ell$-th symbol can be presented as Eq. 1:

$$
\mathrm{X}^{\ell}=\left\{\mathrm{x}^{\ell}[0] \ldots \mathrm{x}^{\ell}[\mathrm{N}-1]^{\mathrm{T}}=\frac{1}{\sqrt{\mathrm{N}}} \sum_{\mathrm{k}=0}^{\mathrm{N}-1} \mathrm{~S}_{\mathrm{k}}^{\ell} \mathrm{e} \frac{12 \pi \mathrm{kn}}{\mathrm{N}}, \ell=0 \ldots \infty\right.
$$

where, $\mathrm{N}$ denotes the number of sub-carriers and $\mathrm{S}_{\mathrm{k}}^{\ell}$ is the frequency-domain complex base-band symbol modulated on the k-th sub-carrier at OFDM symbol $\ell$. The classical metric to evaluate the Peak Average Power Ratio (PAPR) is defined as Eq. 2:

$$
\operatorname{PAPR}(X)=10 \log _{10}\left(\frac{\|X\|_{\infty}^{2}}{E\left(\|X\|_{2}^{2}\right) / N}\right) d b
$$

where, $\mathrm{E}($.$) is the expectation and \|\mathrm{x}\|_{\infty}$ and $\|\mathrm{x}\|_{2}$ represent the $\infty$-norm and the 2-norm of $\mathrm{x}$, respectively.

\subsection{The Proposed PSO-ACE-POCS Algorithm Applied to STBC MIMO-OFDM System}

The simplest $2 \times 2$ STBC with full diversity was first introduced by Alamouti. The encoder takes a block of two QAM symbols $\mathrm{X}_{1}$ and $\mathrm{X}_{2}$ and assigns it to the two transmit antennas according to the following code matrix Eq. 3:

$\mathrm{X}_{\mathrm{A}}=\left[\begin{array}{cc}\mathrm{X}_{1} & \mathrm{X}_{1}^{*} \\ \mathrm{X}_{2} & -\mathrm{X}_{2}^{*}\end{array}\right]$

where, the i-th row represents the i-th transmission period and the $\mathrm{j}$-th column corresponds to the $\mathrm{j}$-th antenna's symbols. The solution to the PAPR reduction problem can be obtained with a Projection onto Convex Sets method, where only one generate population iteration in PSO process was needed to considerably reduce the PAPR. Due to the structure of orthogonal space-time block codes, the SISO ACE-POCS algorithm need only be applied for the first transmission period, since it can be proven that complex sequences $\mathrm{x}_{\mathrm{i}}$ and $\pm \mathrm{X}_{\mathrm{i}}^{*}$ exhibit the same PAPR properties. Therefore, we only need to perform ACE-POCS processing on each SISO block independently, obtaining the minimum PAPR time-domain signal ready to transmit after adding the cyclic prefix for the first transmission period. To obtain the time-domain signals for the rest of the transmission periods, the scaling and conjugate DFT properties can be used to avoid the unnecessary IFFT operations for the remaining transmission periods across all transmit antennas.

The main idea of the ACE-POCS algorithm is to look at the residual clipped-off signal and project it onto the space of allowable extension vectors. The result is a peak reduction signal scaled by an optimal step size subtracted from the time domain signal. For STB block codes, to correctly decode the signal, we project the residual clipped-off signal into the space of allowable STBC extensions, which depends on the code being used.

In general, the set of possible ACE vectors will be convex and a POCS-based algorithm similar to that of (Ouqour et al., 2010) can be formulated to achieve the optimal solution for minimizing a peak below some amplitude. The two convex sets are:

- The set $\mathrm{S}_{\mathrm{A}}$ consisting of all vectors $\mathrm{y} \in \mathrm{R}^{\mathrm{N}}$ such that $|\mathrm{y}|_{\infty} \leq \mathrm{A}$ for some positive constant $\mathrm{A}$ 
- The set $\mathrm{S}_{\mathrm{c}}$, an $\mathrm{N}$-dimentional subspace of $\mathrm{R}^{\mathrm{N}}$, consisting of all vectors $y$ with FFT Y that satisfy the data-dependant ACE restriction

Below is a basic pseudocode for an STBC OFDM system based on ACE-POCS method:

- Starting with the input bit stream, break it up into blocks of size $\mathrm{N}$ for parallel transmission, where there are $\mathrm{N}$ subchannels total. Consider transmission of $\mathrm{p}$ OFDM blocks per antenna. Perform QPSK or 16-QAM modulation to obtain the frequencydomain symbols, $\mathrm{X}_{\mathrm{k}}$

- Add transmit diversity by performing space-time block coding using an STBC and determine the allowable extensions for each subchannel

- Take the IFFT for each antenna's signal to construct the time signal, $\mathrm{x} 0[\mathrm{n}, \ell]_{\mathrm{t}}$, for each antenna $\ell$ (n is the subchanne $\ell$ index, $\ell$ is the antenna index and $t$ is the transmission period index). Set $i=0$

- Clip any $\mid x[n] \geq A$, in magnitude (i.e., project onto $\mathrm{S}_{\mathrm{A}}$ ) to obtain Eq. 4 and 5:

$$
\bar{x}[n]=\left\{\begin{array}{c}
x[n],|x[n]| \leq A \\
A e^{J \varnothing[n]},|x[n]|>A
\end{array}\right.
$$

Where:

$$
x[n]=|x[n]| e^{J \oslash[n]}
$$

- Obtain via $\bar{X}$ an FFT applied to $\bar{x}$

- Enforce all ACE constraints (i.e., project onto $\mathrm{S}_{\mathrm{c}}$ ) on $\overline{\mathrm{X}}$ by restoring all interior points to their original values, while projecting exterior points into the region on increased margin

- Apply an FFT to each antenna's clipped signal to obtain Cclip[k, 1 $]_{\mathrm{t}}$

- Apply PSO algorithm as presented below to $\mathrm{C}_{\text {clip }}$ vector using one generate population $(\mathrm{G}=1)$

Keep only the components of $\mathrm{C}_{\text {clip }}[\mathrm{k}, 1]_{\mathrm{t}}$ which are acceptable extension directions for the given subchannel constellations and set all remaining elements to zero (i.e., project onto $\mathrm{S}_{\mathrm{C}}$ ).

Apply the STBC constraints (i.e., project onto $\mathrm{S}_{\mathrm{B}}$ ) by taking the maximum ACE extension and enforcing it on each block according to the code matrix. (Note that this step ensures that the STBC constraints are still satisfied; thus the optimal decoding methods associated with the STBC are still optimal).

\section{Apply an IFFT to obtain c $[\mathrm{n}, 1]_{\mathrm{t}}$.}

\subsection{The Particle Swarm Optimization}

The PSO is a population based stochastic optimization technique; system is initialized with a population of random solutions and searches for optima by updating generations. However, PSO has no evolution operators such as crossover and mutation. In PSO, the potential solutions, called particles, fly through the problem space by following the current optimum particles with respect to a specific algorithm. In a previous works (Ouqour et al., 2010; 2011a; Wen et al., 2008) Active Constellation Extention Approximate Gradient Project (ACE-AGP) combined with PSO and a suboptimal Partial Transmit Sequence (PTS) based on PSO algorithms have been presented for the low computation complexity and the reduction of the PAPR of an OFDM signal.

The procedure of standard PSO can be summarized in Fig. 2.

In this study, during the PSO process (Ouqour et al., 2011b), each potential solution is represented as a particle with a position vector $\mathrm{C}_{\text {clip }}$, referred to as clipped-off portion and a moving velocity represented as $\mathrm{C}_{\text {clip }}$ and $\mathrm{V}$, respectively.

Thus for a K-dimensional optimization, the position and velocity of the $\mathrm{i}$-th particle can be represented as Eq. 6 :

$$
\mathrm{C}_{\text {clip }}=\left(\mathrm{C}_{\text {clip }}{ }_{\mathrm{i}}^{1}, \mathrm{C}_{\text {clip }} \stackrel{2}{\mathrm{i}}, \ldots . \mathrm{C}_{\text {clip }}{ }_{\mathrm{i}}^{\mathrm{k}}\right) \text { and } \mathrm{v}_{\mathrm{i}}=\left(\mathrm{v}_{1}^{\mathrm{i}}, \mathrm{v}_{2}^{\mathrm{i}}, \ldots . . \mathrm{v}_{\mathrm{k}}^{\mathrm{i}}\right)
$$

Respectively each particle has its own best position Eq. 7:

$$
\mathrm{C}_{\text {clip }} \frac{\mathrm{p}}{\mathrm{i}}=\left(\mathrm{C}_{\text {clip }}{ }_{\mathrm{i}}^{1}, \mathrm{C}_{\text {clip }} \stackrel{2}{\mathrm{i}}, \ldots . . \mathrm{C}_{\text {clip }} \mathrm{k}\right)
$$

Corresponding to the individual best objective value obtained so far at time $t$, referred to as pbest. The global best (gbest) particle is denoted by Eq. 8:

$$
\mathrm{C}_{\text {clip }}{ }^{\mathrm{G}}=\left(\mathrm{C}_{\text {clip }} \stackrel{\mathrm{g}}{1}, \mathrm{C}_{\text {clip }} \stackrel{\mathrm{g}}{2}, \ldots . \mathrm{C}_{\text {clip }} \underset{\mathrm{k}}{\mathrm{g}}\right)
$$

Which represents the best particle found so far at time $t$ in the entire swarm. The new velocity $V_{i}(t+1)$ for particle $\mathrm{i}$ is updated by Eq. 9:

$$
\begin{aligned}
& \mathrm{V}_{\mathrm{i}}(\mathrm{t}+1)=\mathrm{w} * \mathrm{~V}_{\mathrm{i}}(\mathrm{t})+\mathrm{c}_{1} * \mathrm{r}_{1} * \mathrm{C}_{\text {clip }} \mathrm{p}_{\mathrm{i}}^{\mathrm{p}}-\mathrm{C}_{\text {clip }_{\mathrm{i}}}(\mathrm{t}) \\
& +\mathrm{c}_{2} * \mathrm{r}_{2} * \mathrm{C}_{\text {clip }}{ }^{\mathrm{G}}(\mathrm{t})-\mathrm{C}_{\text {clip }}(\mathrm{t})
\end{aligned}
$$




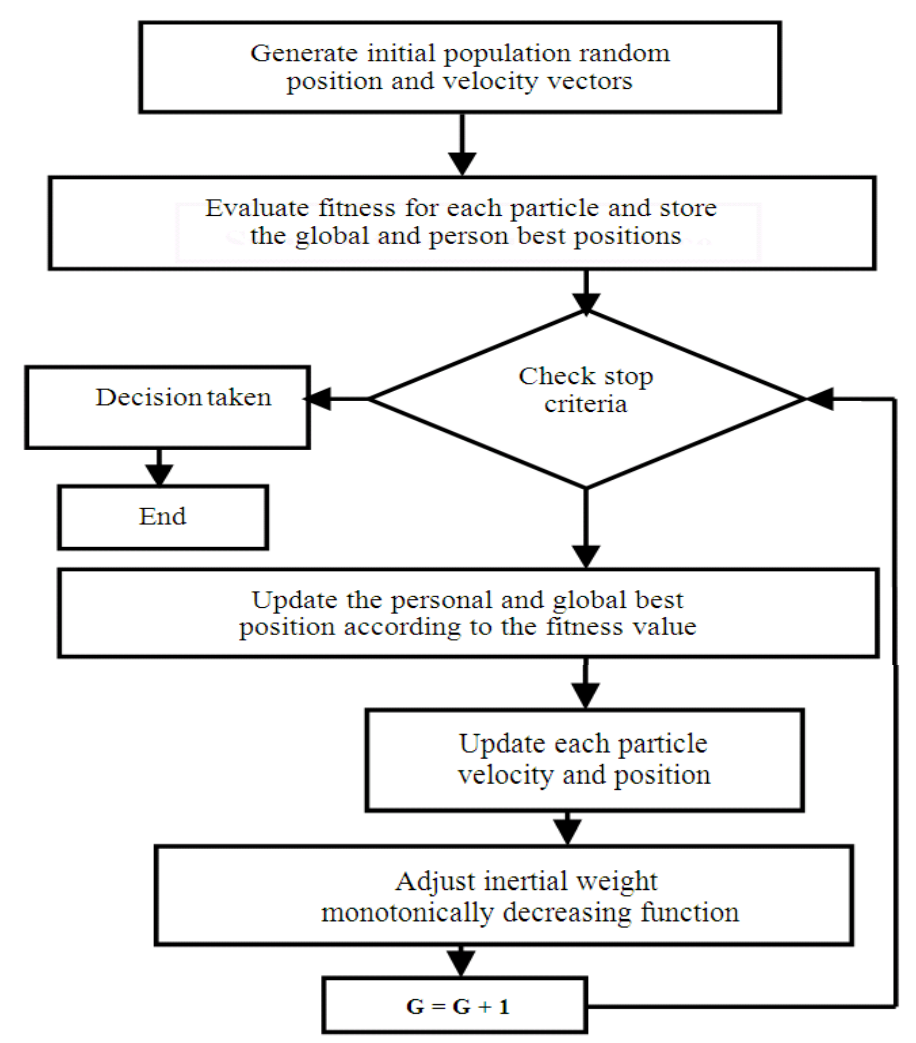

Fig. 2. PSO algorithm

where, $\mathrm{V}_{\mathrm{i}}(\mathrm{t})$ is the old velocity of the particle $\mathrm{i}$ at time $\mathrm{t}$. Apparent from this equation, the new velocity is related to the old velocity weighted by weight $\mathrm{w}$ and also associated to the position of the particle itself and that of the global best one by acceleration factors $c_{1}$ and $c_{2}$. The $c_{1}$ and $c_{2}$ are therefore referred to as the cognitive and social rates, respectively, because they represent the weighting of the acceleration terms that pull the individual particle toward the personal best and global best positions. The inertia weight $\mathrm{w}$ in Eq. 11 is employed to manipulate the impact of the previous history of velocities on the current velocity. Generally, in population-based optimization methods, it is desirable to encourage the individuals to wander through the entire search space, without clustering around the local optima, during the early stage of the optimization.

A suitable value for $\mathrm{w}(\mathrm{t})$ provides the desired balance between the global and local exploration ability of the swarm and, consequently, improves the effectiveness of the algorithm. Experimental results suggest that it's preferable to initialize the inertia weight to a large value, giving priority to global exploration of the search space, linear decreasing $\mathrm{w}(\mathrm{t})$ so as to obtain refined solutions (Clerc and Kennedy, 2002) and 19 (Shi and Eberhart, 1998). For the purpose of intending to simulate the slight unpredictable component of natural swarm behavior, two radôme functions $r_{1}$ and $r_{2}$ are applied to independently provide uniform distributed numbers in the range $[0,1]$ to stochastically vary the relative pull of the personal and global best particles.

Based on the updated velocities, new position for particle $\mathrm{i}$ is computed according the following Eq. 10:

$\mathrm{c}_{\text {clip }}(\mathrm{t}+1)=\mathrm{c}_{\text {clip }}(\mathrm{t})+\mathrm{V}(\mathrm{t}+1)$

The populations of particles are then moved according to the new velocities and locations calculated by (9) and (10) and tend to cluster together from different directions (Wen et al., 2008). Thus, the evaluation of each associate fitness of the new population of particles begins again. The algorithm runs through these processes iteratively until it stops. In this study, the current position can be modified by Eq. 11: 
$\mathrm{w}(\mathrm{t})=\mathrm{w}_{\max }-\left(\mathrm{w}_{\max }-\mathrm{w}_{\min }\right) * \mathrm{iter}_{\text {iter }_{\max }}$

Where:

$\mathrm{W}_{\max }=$ The initial weight

$\mathrm{w}_{\min }=$ The final weight

iter $_{\max }=$ Maximum number of iterations and iter is the current iteration number

\section{RESULTS}

The results of the ACE-POCS algorithm applied to STBC MIMO-OFDM are shown in Fig. 3. The algorithm was tested for a complex-baseband STBC OFDM system for two-antennas when $\mathrm{N}=256$ and $\mathrm{N}=512$ subchannels employing QPSK and 16-QAM using $2^{13}$ randomly generated OFDM symbol blocks. As shown in Fig. 1 for Alamouti STBC, the tenth iteration achieves an important PAPR reduction. Table 1 below summarizes the PAPR reduction gains obtained using 256 and 512 subcarriers with QPSK and 16-QAM modulations for MIMO-OFDM system added to STBC when looking at a $10^{-4}$ symbol clip probability.

Figure 4 evaluates the BER performance of a $2 \times 2$ STBC MIMO-OFDM system under QPSK modulation and ACE-POCS, assuming Additive White Gaussian Noise (AWGN) conditions. As usual, the combination of PSO ACE-POCS and STBC results in improved noise immunity than independently used ACE-POCS added to STBC.

Table 1. PAPR Reduction results under different number of subcarriers and modulation types for two antennas

\begin{tabular}{|c|c|c|c|}
\hline Modulation & Subcarriers & $\begin{array}{l}\text { STBC ACE-POCS } \\
\text { Iteration }=10 \\
\Delta \mathrm{p} 1\end{array}$ & $\begin{array}{l}\text { STBC PSO-ACE-POCS } \\
\text { Iteration }=10 \\
\Delta \mathrm{p} 2\end{array}$ \\
\hline \multirow[t]{2}{*}{$\overline{\text { QPSK }}$} & 256 & $4.2 \mathrm{db}$ & $6.4 \mathrm{db}$ \\
\hline & 512 & $4.9 \mathrm{db}$ & $6.6 \mathrm{db}$ \\
\hline \multirow[t]{2}{*}{ 16-QAM } & 256 & $3.4 \mathrm{db}$ & $5.4 \mathrm{db}$ \\
\hline & 512 & $3.5 \mathrm{db}$ & $5.8 \mathrm{db}$ \\
\hline
\end{tabular}
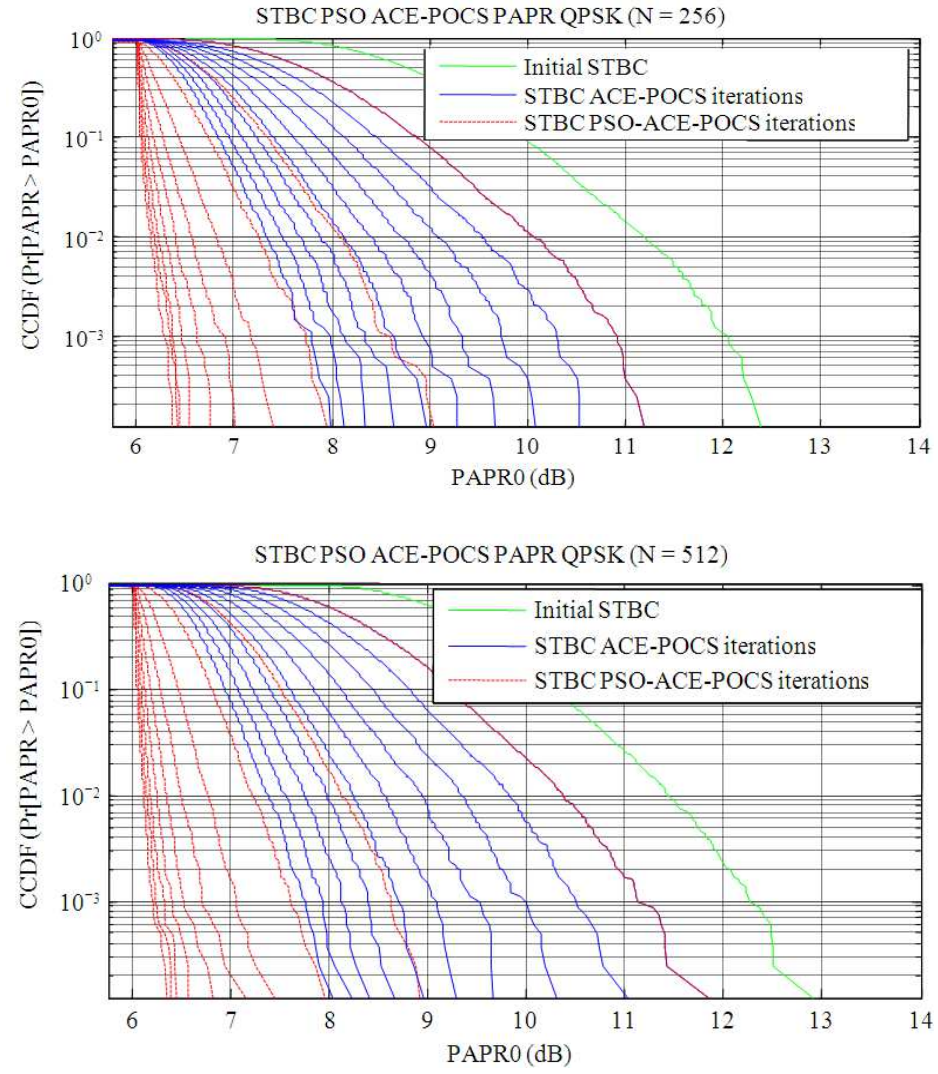
Ahmed Ouqour et al. / Journal of Computer Science 8 (12) (2012) 2017-2024
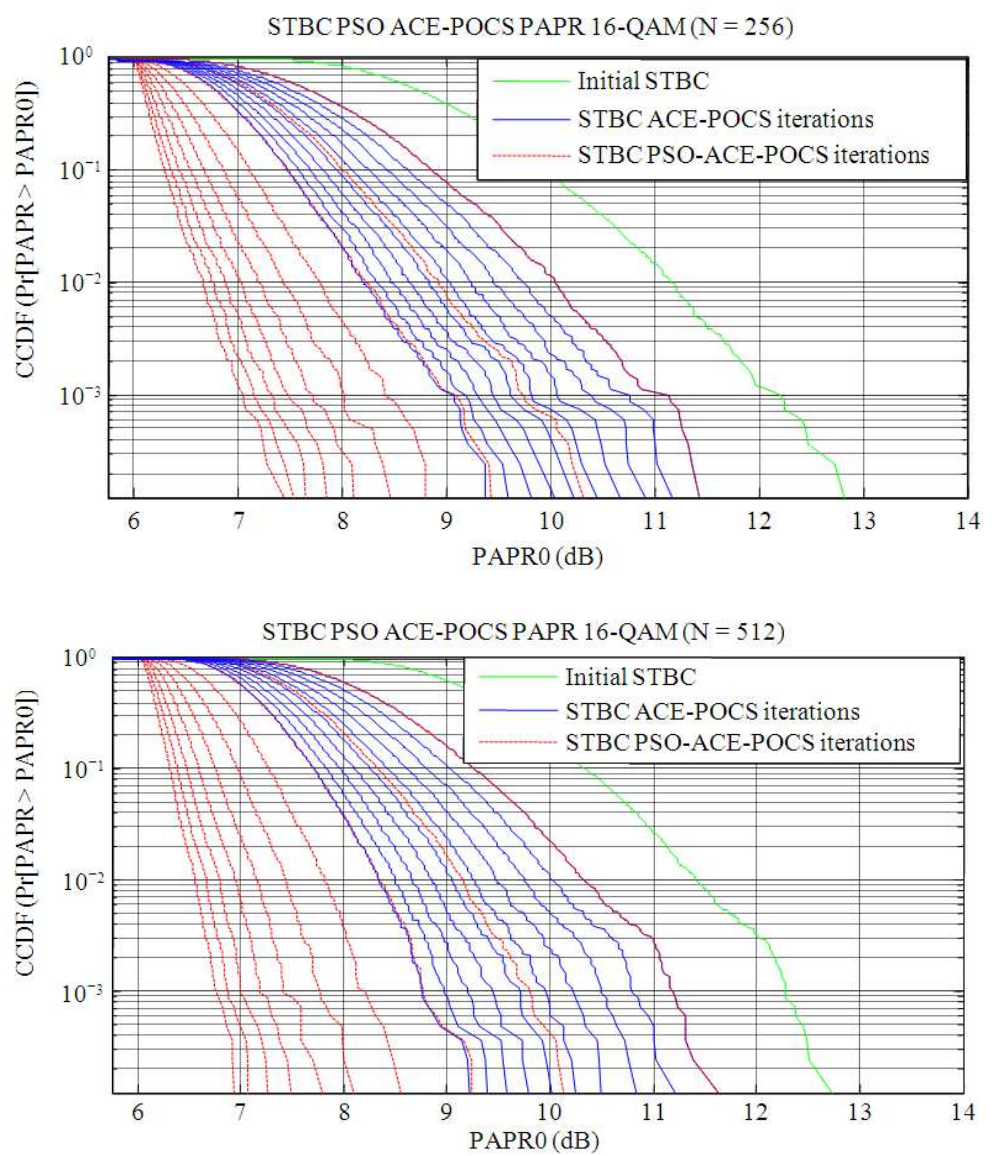

Fig. 3. Comparison of PAPR reduction between ACE-POCS and PSO-ACE-POCS methods applied to STBC MIMO-OFDM system with 256 and 512 subchannels employing QPSK and 16-QAM modulations

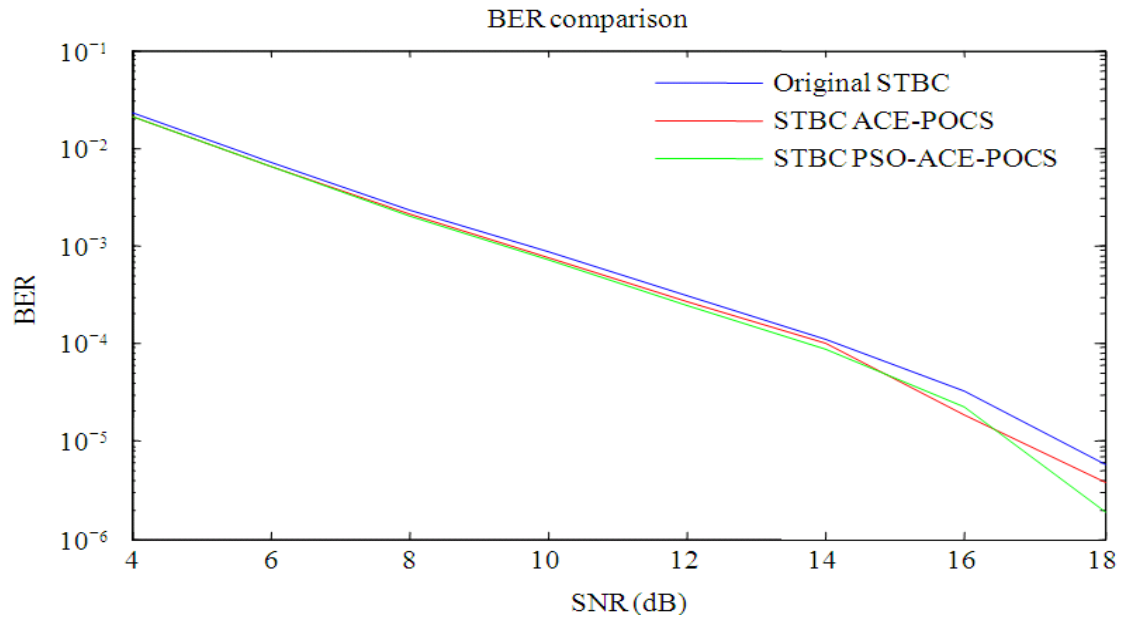

Fig. 4. BER performance results with 2-Tx antennas STBC using 256 subcarriers employing QPSK under AWGN conditions in MIMO-OFDM system 


\section{DISCUSSION}

Our proposed method based on PSO can reduce the PAPR more than the original STBC using ACE-POCS method by $\sim 2.2 \mathrm{db}, \sim 1.7 \mathrm{~dB}$ respectively for $\mathrm{N}=256, \mathrm{~N}$ $=512$ when employing QPSK modulation and by $\sim 2 \mathrm{db}$, $\sim 2.2 \mathrm{~dB}$ respectively for $\mathrm{N}=256, \mathrm{~N}=512$ when employing 16-QAM modulation.

Note that the PAPR reduction in the third iteration for our proposed method using PSO is much better than the PAPR reduction in the tenth iteration for the ACE-POCS method which do not use PSO.

It is worth noting that these results are achieved with low complexity and with only one generate population $(\mathrm{G}=1)$ in PSO process.

\section{CONCLUSION}

In this study, the proposed algorithm for reducing the envelope fluctuations in MIMO-OFDM system has been investigated. Simulation results show that, this algorithm is able to obtain signals with low envelope fluctuations with very limited complexity and this is valid for any number of sub-carriers for both QPSK and 16-QAM modulations. Moreover, it achieves better performances than previous work. It is worth noting that this study can be exploited in real implementation.

\section{REFERENCES}

1. Alamouti, S.M., 1998. A simple transmit diversity technique for wireless communications. IEEE J. Select. Areas Comm., 16: 1451-1458. DOI: 10.1109/49.730453

2. Beek, J.J.V.D., O.S. Edfors, M. Sandell, S.K. Wilson and O.P. Borjesson, 1995. On channel estimation in OFDM systems. Proceedings of the IEEE 45th Vehicular Technology Conference, Jul. 25-28, IEEE Xplore Press, Chicago, IL., pp: 815-819. DOI: 10.1109/VETEC.1995.504981

3. Clerc, M. and J. Kennedy, 2002. The particle swarm-explosion, stability and convergence in a multidimensional complex space. IEEE Trans. Evolut. Comput., 6: 58-73. DOI: 10.1109/4235.985692

4. Davis, J.A. and J. Jedwab, 1999. Peak-to-mean power control in OFDM, golay complementary sequences and Reed-Muller codes. IEEE Trans.
Inform. Theory, 45: 2397-2417. DOI: 10.1109/18.796380

5. Gesbert, D., M. Shafi, D.S. Shiu, P.J. Smith and A. Naguib, 2003. From theory to practice: An overview of MIMO space-time coded wireless systems. IEEE J. Selected Areas Commun., 21: 281-302. DOI: 10.1109/JSAC.2003.809458

6. Ouqour, A., Y. Jabrane, B.A.E Said and A.A. Ouahman, 2010. Active constellation extension combined with particle swarm optimization for PAPR reduction in OFDM signals. Int. J. Comput. Network Secu., 2: 43-47. http://www.doaj.org/doaj?func=abstract\&id=64 4302

7. Ouqour, A., Y. Jabrane, B.A.E. Said and A.A. Ouahman, 2011a. Particle swarm optimization for PAPR reduction in OFDM signals with modified pilots. Proceedings of the International Conference on Multimedia Computing and Systems, Apr. 7-9, IEEE Xplore Press, Ouarzazate, $\quad$ pp: 1-3. DOI: 10.1109/ICMCS.2011.5945718

8. Ouqour, A., Y. Jabrane, B.A.E. Said and A.A. Ouahman, 2011b. Performance comparison in OFDM signals between PTS and ACE based on particle swarm optimization. Proceedings of the 2nd International Workshop on Codes, Cryptography and Communication Systems, (CCCS' 11), pp: 16-17.

9. Shi, Y. and R. Eberhart, 1998. A modified particle swarm optimizer. Proceedings of IEEE International Conference on Evolutionary Computation, May 4-9, IEEE Xplore Press, Anchorage, AK., pp: 69-73. DOI: 10.1109/ICEC.1998.699146

10. Tsiligkaridis, T. and D.L. Jones, 2010. PAPR reduction performance by active constellation extension for diversity MIMO-OFDM systems. J. Elect. Comput. Eng., 2010: 5-5. DOI: 10.1155/2010/930368

11. Wen, J.H., S.H. Lee, Y.F. Huang and H.L. Hung, 2008. A suboptimal PTS algorithm based on particle swarm optimization technique for PAPR reduction in OFDM systems. J. Wireless Commun. Network., 2008: 1-8. DOI: $10.1155 / 2008 / 601346$

12. Yang, H., 2005. A road to future broadband wireless access: MIMO-OFDM-based air interface. IEEE Commun. Mag., 43: 53-60. DOI: 10.1109/MCOM.2005.1381875 